bienn ist, jedenfalis dass die Rosette bereits im Herbst vorgebildet ist. Stengelbildung fehlt meist ganz.

Die Farbe ist lila-fleischfarbig, etwa wie unser Pap. somniferum der Giirten. Die Blattfärbung jst wio bei $P$. arenarium, dem unsere Pflanze wohl am nächsten steht; aber Narbenstrahlen nur 4-5 (mehr 6-9). Mit $C$. laevigatum var, erosum lässt sich eben deshalb die Pflanze nicht in Verbindung bringen, auch ist der Basalfleck tatsächlich an der Basis und die Blattgestalt und Behaarung spricht auch gegen eine Vereinigung."

\title{
XVII. Cyperaceae novae. III. ${ }^{1}$ )
}

Von G. Kükenthal-Coburg.

(Originaldiagnosen.)

15. Kyllingia alba Nees var. diminuta Kükenth., nov. var.

Culmus nonnisi $5-10 \mathrm{~cm}$ altus gracilior quam in forma typica. Spicae 3 breviores $5-7 \mathrm{~mm}$ longae. Spiculae $2^{1 / 2} \mathrm{~mm}$ longae. Squamae epunctatae obtusiores.

Kamerun: Garua, sandige steinige Gebüschsavane, $300 \mathrm{~m}$ (C. L.edermann no. 3279!).

16. Kyllingia odorata Vahl var, bulbifera Kükenth., nov. var.

Culmi basi bulboso-incrassati.

Argentinien: Siambon (Lorentz no. 197!).

17. Kyllingia erecta Schumacher (=K. Soyauxii Boeck.) var. Schlechteri Kükenth.

K. brevifolia C. B. Clarke in Dyer, Fl. trop. Afr., VIII (1902), 273 p. p., non Rottb.

Folia nonnisi $1-2$ breviter acuminata. Squamae carina valde et grosse spinulosae. Nux oblonga.

Congostaat: Irebu (Schlechter no. 12612!); Sicia am unteren Congo (Dupuis no. 21 !).

18, Kyllingia erecta var, pleiocarpa Kükenth., nov, var.

Spiculae triflorae nuces 2 producentes. Nux oblonga.

Togo: Steppe bei Paratau (R. Büttner no. 626 !).

19. Kyllingia erecta var. intercedens Kükenth., nov. var.

Culmus strictus elatus rigidior. Squamae aureo-tinctae. Nux obovata.

Ny assaland: Mt. Zomba, 4000-6000' (Why te!).

20. Kyllingia erecta var. aurata (Nees) Kükentb.

$K$. aurata Nees in Linnaea X (1835-36), 139.

Culmi $4-20 \mathrm{~cm}$ alti subincurvi. Folia $1-1 \frac{1}{2} \mathrm{~mm}$ lata complicatoplana longe acuminata. Squamae aureo-ferrugineae. Nux oblonga.

Süd afrika.

1) I. cf. Rep. VIII (1910), p. 7-8; II. cf. VIII (1910), pp. 326-327. 
21. Kyllingia peruciana Lam. var. foliata Kükenth., nov. var.

Vaginae nonnullae foliatae. Bractede spicam paullo superantes.

Jamaica: Ocho Rios (N. L. Britton et Arthur Hollick no. 2705!).

22. Kyllingia melanosperına Nees var. plurifoliata Kükenth, nov. var.

Folia 2-3 evoluta. Spiculae oblongo-lanceolatae $4 \mathrm{~mm}$ longae plerumque uniflorae.

II dagascar: In Sümpien bei Betsiléo (Hildebrandt no. 4019 !).

23. Fyllingia pulchella Kunth forma robustior Kükenth., nov. form.

Culmi validiores. Folia latiora. Spicae 3 longiores latioresque, centralis ad $14 \mathrm{~mm}$ longa oblonga. Spiculae $4 \mathrm{~mm}$ longae.

Abysinien: Gottes Claudius 7500' (Schimper no. 1313!); Galat (sichimper no. 1315 !).

24. Kyllingia chrysantha K. Schum. var, decolorans Kükenth., nov. var.

Squamae decolorantes sordide stramineae obsoletissime nervosae.

Deutsch-Ost-Afrika: Hochplateau von Lhehe bei Iringa (Magdal. Prince!).

25. Kyllingia platyphylla K. Schum. rar. Iongifolia Kükenth., nov. var.

Folia bracteaeque longiora.

Portugiesisch-Ost-Afrika: Luia, Busch in der Nähe des Luiaflusses (W. Tiesler no. 20 p. p. ').

26. Kyllingia leucocephala Boeck. var. pluriceps Kükenth., nov. var.

Spicae 3-5. Spiculae nucem unicam producentes. Squamae albovirides.

Kamelun: Station Garua bei Schuari, $300 \mathrm{~m}$ (Ledermann no. 4612 p. p.'?.

27. Cyperus (Fycreus) muricatus Kükenth.

Pycreus Rehmannianus C. B. Clarke in Dyer, Fl. trop. Afr., VIII (1902), 291, non in Dyer. Fl. capens.

Radix fibrosa. Culmus $30-50 \mathrm{~cm}$ altus strictus tirmus triangularis laevis basi paucifoliatus. Folia culmo breviora $2-2^{1} / 2 \mathrm{~mm}$ lata, vaginae rufescentes. Anthela laxa 5--7-radiata, radii laterales ad $5 \mathrm{~cm}$ longi. Bracteat 3-4 patentes, inferiores anthelam superantes. Spiculae laxe spicatae lineari-oblongae $10-18 \mathrm{~mm}$ longae fere $3 \mathrm{~mm}$ latae patentes compressae acutae ad 30-florae. Squamae dense imbricatae lanceolatoovatae subobtusae fusco-sanguineae vel brunneae marginibus anguste hyalinae lateribus subsulcatae dorso carinato viridi tricostatae. Nux $1 / 3$ squamae aequans $3 / 4 \mathrm{~mm}$ longa obovata biconvexa turgidula basi in stipitem latum contracta apiculata rufa transversim nuricata (cellulae extimae oblongae). Stamina 3. Stigmata 2.

Deutsch-Ost-Afrika: ïstliche Ausläufer des Mampqui-Rückens (W. Busse no. 709 !). Britisch-Central-Afrika: Nyassaland, Mt. Zomba, $4000-6000^{\prime}$ (A. Whyte!).

Durch die Form der Nuss von Cyperus Rehmannianus leicht zu scheiden und Cyperus pauper und C. diculsus genähert. 
28. Cyperus (Pycreus) latespicatıs Boock. var. gracilescens Kükenth., nov. var.

Culmus tenuis. Folia perangusta. Spiculae nonnisi $3 \mathrm{~mm}$ latae.

Süd-Indien: Shimoga Maisor (A. Meobold no. 9353!).

29. Cyperus (Pycreus) zonatus Kïkenth, nov. spec.

Radix fibrosa, fibrillis numerosis capillaribus. Culmus $2-4 \mathrm{~cm}$ altus pertenuis debilis compresso-triangularis profunde sulcatus laevis inferne paucifoliatus. Folia brevia perangusta complicala longe vaginantia, vaginae brunneae. Bracteae 2 inaequales, ima culmum quasi continuans, anthelam superantes. Anthela simplex $1-3$ radiata, radii subsessiles vel parum elongati 3-7-stachyi. Spiculae oblique patentes satis dense spicatae lineares $4-10 \mathrm{~mm}$ longae $1 \mathrm{~mm}$ latae $12-20$-florae. Rhacheola recta exalata, foveis profundis oblongis. Squamae subdense imbricatae ovatae obtusiusculae sordide stramineace carina tricostatae. Nux 1/3 squamae aequans oblongo-ellipsoidea biconvexa brunnea albo-undulata apice obtusa. Stylus bifidus. Stamina 2.

Congostaat: Stanley Pool (Schlechter no. 12577!).

Gehört in die Verwandtschaft von Cyp. flavescens L.

30. Cyperus (Pycreus) acuticarinatus Kükenth., nov. spec.

Radix fibrosa. Culmus ad $55 \mathrm{~cm}$ usque altus firmulus compressotriangularis laevis inferne foliatus. Folia culmo breviora $2 \mathrm{~mm}$ lata carinato-complicata rigidula, vaginae imae fuscescentes dissolutae. Bracteae 2-3 patentes, ima anthelam superans. Anthela satis contracta 4-radiata, radii laterales breves vel divergentes lineares $8-12 \mathrm{~mm}$ longae $2 \mathrm{~mm}$ latae compressae acutae ad 30-florae. Squamae spissae ovatae acutae brunneae marginibus anguste albo-hyalinae e carina peracuta tricostata viridi mucronatae. Nux $1 / 3$ squamae aequans late obovata turgido-biconvexa basi cuneata conspicue apicata rufa dense punctata. Stigmata 2. Stamina 3, antherae rubrae lineares.

Togo: Sokode, an feuchten Wiesenstellen (Schilling no. 6!).

Von dem naheverwandten Cyperus globosus All. hauptsächlich durch die scharfgekielten spitzen Schuppen und die breitere beiderseits geschwollene Nuss getrennt. Die Zahl der Staubfäden beträgt 3 , bei $C$. globosus 2.

31. Cyperus (Pycreus) lanceus Thunb. var. divaricatus Kükenth., nov. var.

Culmus $15 \mathrm{~cm}$ altus subincurvus. Rami anthelae divaricati paucistachyi. Spiculae angustiores acutissimae. Squamae purpurascentes. Stigmata valde exserta.

Central-Madagascar: Antananarivo (Rutenberg!); Andrangolóaka (Hildebrandt no. 3743 b!).

32. Cyperus (Pycreus) longivaginans Kükenth., nov. spec.

Perennis. Culmus $30-45 \mathrm{~cm}$ altus firmus trigonus latere uno profunde sulcatus foliis 2-3 remotis obsitus. Folia culmum subsuperantia perangusta semiteretia coriacea pungentia, vaginae longissimae rufescentes, Bracteae 3 inaequales semiteretes, inferiores anthelam longe 
superantes. Anthela sublaxa subsimplex $3-5$-radiata, radii onnes pedunculati ad $2^{1 / 2} \mathrm{~cm}$ longi erecto-patentes saepe basi ramosi ex ocreis ampliatis oblique sectis orti pluristachyi. Spiculae demum patentes linearilanceolatae acutae compressae $10-14$-florae $6-8 \mathrm{~mm}$ longae $1 \frac{1}{2} \mathrm{~mm}$ latae. Squamae dense imbricatae lanceolato-ovatae acutiusculae atrofuscae nitidae marginibus imprimis apicem versus sordide albidae carina straminea tricostatae. Nux non evoluta. Stamina 3.

Central-Madagascar; Ost-Imerina, Andrangolóaka im Sumpf (Hildebrandt no. 3736!).

Boeckeler hielt diese Art für Cyperus atro-brunneus Baker. Sie steht jedoch Cyperus Cooperi C. B. Clarke weit rähor, von welcher sie durch lockere Spirre und weissberandete Schuppen abweicht.

33. Cyperus (Pycreus) nigricans Steud. var. firmior Kükenth., nov. var.

Culmus firmus triqueter. Folia coriacea sicut bracteae pungentia. Anthela magis compacta. Nux obovato-oblonga evidentius apicata.

Leutsch-ost-Afrika: Lluguru (Stuhlmann no. 915D!); Kilimandjatro (Volkens no. 2014!); No. Kiwu, Sabyino-Kahinga-Sattel (Mildbraed no. 1761!). Gallahochland: Sidamo (Ellenbeck no. 1861!).

34. Heleocharis dunensis Kükenth., nov. spec.

Rhizoma lignosum descendens pluriceps. Culmi numerosi $15-30 \mathrm{~cm}$ alti tenues obsolete angulati sulcati basi vaginis perangustis longis, suprema inferne purpurea superne pallide brunnea ore ferrugineo truncata postice breviter producta, obsiti. Spicula oblonga obtusa $7-9 \mathrm{~mm}$ longa $21 / 2 \mathrm{~mm}$ lata multiflora. Squamae paucispirae oblongo-ovatae vel inferiores ovatae obtusae carinato-naviculares fusco-castaneae marginibus anguste albo-byalinae carina stramineae. Nux parvula obovata tumidotriangularis angulis prominentibus cancellata olivacea nitida. Stylus rigidulus trifidus basi pyramidali pallida triangulari inferne triloba incumbens. Stamina 3. Setae hypogynae nullae vel rudimentariae.

Uruguay: Dep. Maldenado. Piriapolis, in dunis locis udis (Corn. Osten no. 5716!).

Von Heleocharis chaetaria Roem. et Schult. durch höheren Wuchs, längere schmalere Infloreszenz und glänzende Nuss, von $H$. arenaria lienth. durch das abwärts gerichtete Rhizom und die engen Basalscheiden getrennt.

35. Scirpus Forsythii Kükenth., nov. spec.

Rhizoma repens tenue. Culmi plures $5-10 \mathrm{~cm}$ alti stricti vel leviter incurvi firmuli obsolete trigoni inferne vaginis brunneis oblique sectis aphyllis cincti. Spicula unica terminalis $6-8 \mathrm{~mm}$ longa oblongo-ellipsoidea bractea erecta $1 \frac{1}{2} \mathrm{~cm}$ longa culmum quasi continuante suffulta. Squamae membranaceae oblongo-ovatae subobtusae clare brunneae nitidulae obsolete plurinervosae e carina pallida breviter mucronatae. Nux $1 / 2$ squamae aequans obovata plano-convexa brunnea demum spadicea nitida obsolete rugosa basi in stipitem latum contracta apicata. Setae 4 retrorsum scabrae nucem paullo superantes.

Neu-Süd-Wales: Nepean River (WW. Forsyth!). 
Die Pflanze, welche ich aus dem National-Herbarium von Sidney erhielt, trug die Bestimmung Scirpus debilis Pursh. Das kriechende Rhizom, die schmaleren Ïhrchen und Schuppen unterscheiden sie jedoch hinlänglich von dieser Art.

36. Rhynchospora campanulata Kükenth., nov. spec.

Radix fibrosa. Culmus $18-25 \mathrm{~cm}$ altus strictus firmus triangularis superne parce pilosus basi plurifoliatus sursum foliis 2 remotis obsitus. Folia culmo breviora $2 \mathrm{~mm}$ lata marginibus revoluta valde carinata longe attenuata carina marginibusque pilosa rigida. Capitula 2 in apice culmi approximata, inferius breviter superius longius pedunculata, bractea erecta $4 \mathrm{~cm}$ longa suffulta, late obconica pluristachya. Spiculae lineari-lanceolatae suberectae. Squamae 7 corjaceae sursum longitudine accrescentes, inferiores 4 vacuae, lanceolatae acuminatae pallidae castaneo-tinctae e carina viridi in aristam longam patentim pilosam excurrentes. Nux $1 / 3$ squamae aequans obovata tumida basi contracta lucide albo-marmorata transversim rugosa apice truncata. Stylus longus indivisus basi campanulato-dilatata albida insidens.

Brasilien: Minas Geraës, Campos bei Ouro Preto (h. Damazio no. 2185 !).

Anscheinend der Rhynchospora aberrans C. B. Clarke sehr nahestehend, abel durch mehrere Merkmale, wie namentlich die mehr genäherten nicht kugeligen, sondern verkehrt-kegelförmigen Köpfchen und die nicht pıramidale sondern glockenförmige Basis des Griffels getrennt.

\title{
XVlII. Sixth Contribution to the Flora of Australia. ${ }^{1}$ )
}

\author{
By K. Dom in (Prague). \\ (Originaldiagnosen.)
}

46. Myoporum latisepalum Domin, nov. spec. (Sectio Chamaepogonia.)

Myoporo debili affine, fruticosum et omnino glabrum, ramulis juvenilibus angulatis postea cylindricis et quoad visum nunquam tuberculatoglandulosis. Folia alternantia in ramulis sat densa brevissime petiolata (basi petioli instar angustata subsessilia) late ovato- vel oblongo-elliptica latitudine circiter duplo longiora ca. $5 \mathrm{~cm}$ longa et $21 / 4-21 / 2 \mathrm{~cm}$ lata obtusa vel obtusissima imo apice acute denticulata caeterum integerrima glaberrima haud coriacea, nervis lateralibus parum prominentibus. Flores plerumque in foliorum axillis bini rarius solitarii, breviter pedicellati; pedicelli calyce breviores; caly $x$ ob lobos latos campanulatus, calycis segmenta foliacea oblonga obtusa et breviter mucronatula sub fructu fere $10 \mathrm{~mm}$ longa et $4 \mathrm{~mm}$ vel paulo plus lata; corolla haud visa;

1) Cf. Rep. XI (1912), pP. $261-264$. 